\title{
Morphology of the Red Rectangle proto-planetary nebula
}

\author{
Nico Koning ${ }^{1}$, Sun Kwok ${ }^{2}$ and Wolfgang Steffen ${ }^{3}$ \\ ${ }^{1}$ University of Calgary, Calgary, Canada \\ email: nico.koning@ucalgary.ca \\ ${ }^{2}$ University of Hong Kong, Hong Kong, China \\ email: sunkwok@hku.hk \\ ${ }^{3}$ Instituto de Astronomia, Universidad Nacional Autonoma de Mexico, Ensenada, Mexico \\ email: wsteffen@astrosen.unam.mx
}

\begin{abstract}
Using the 3D morpho-kinematic modeling software SHAPE, we have created a model of the Red Rectangle that naturally reproduces many exotic morphological features including the notorious "ladder rungs".
\end{abstract}

Keywords. Scattering, stars: AGB and post-AGB

\section{Introduction}

The Red Rectangle (RR) is an unusual proto-planetary nebula (PPN) which was first identified by Cohen et al. (1975). The RR exhibits many singular features which have attracted much attention in recent years (Cohen et al. 2004). Among these include its distinctive "X" shape, the peculiar "ladder rungs" perpendicular to the axis of symmetry and the bright knots (vortices) that seem to connect the ladder rungs to the "X". We present here a simple morphological model of the RR that attempts to explain all three of these features.

\section{Model}

A morphological/radiation transfer model of the RR was developed using SHAPE (Steffen et al. 2011) which can model radiative transfer through gaseous nebulae and simulate scattering. The model (left panel of Figure 1) consists of a central star $\left(T_{\text {eff }}=8250 \mathrm{~K}\right.$ and $\left.L=6000 \mathrm{~L}_{\odot}\right)$ and a bi-conical cavity embedded within a spherical dust halo. We model the Extended Red Emission (ERE) as a scattering species (intensity proportional to density) with custom scattering coefficients. To simulate periodic mass ejections on the AGB, density enhancements in the dust halo where added to the model.

\section{Results}

The center panel of Figure 1 shows the SHAPE rendering of the model. The X shape of the nebula is caused by the geometry of the cavity. The edges are enhanced because it is in this direction that we are looking through the most material. The linear features spanning the bi-cone lobes are a purely optical effect as no such geometry was built into the model. Since the spherical dusty halo has been evacuated within the bi-cones, what remains of the density enhancements (spherical shells) in this region is a thin circular etching of dust on the walls. When seen almost exactly edge on, these etchings appear linear in nature and explain the "ladder rungs" seen in the RR. The bright knots that 


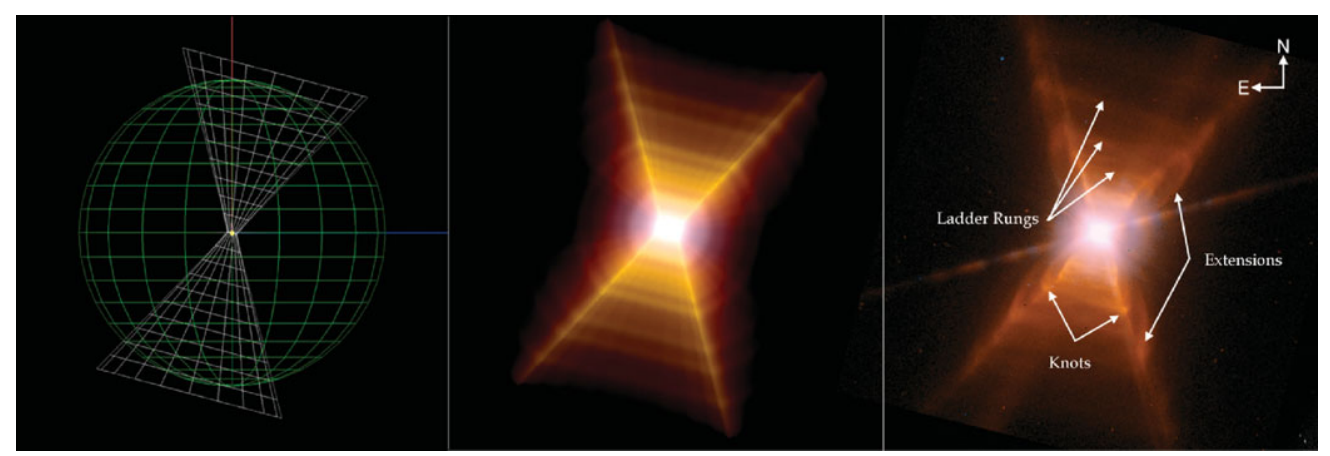

Figure 1. Left: mesh SHAPE model of the RR. Center: SHAPE rendering of the RR with an inclination of $0^{\circ}$. The image was rendered from $400 \mathrm{~nm}$ (blue) to $700 \mathrm{~nm}$ (red). Right: HST image of the RR Nebula. The color scales are the same and both images are displayed in a LOG scale. (HST image credit: PI Van Winckel, GO7297, Hubble Archives).

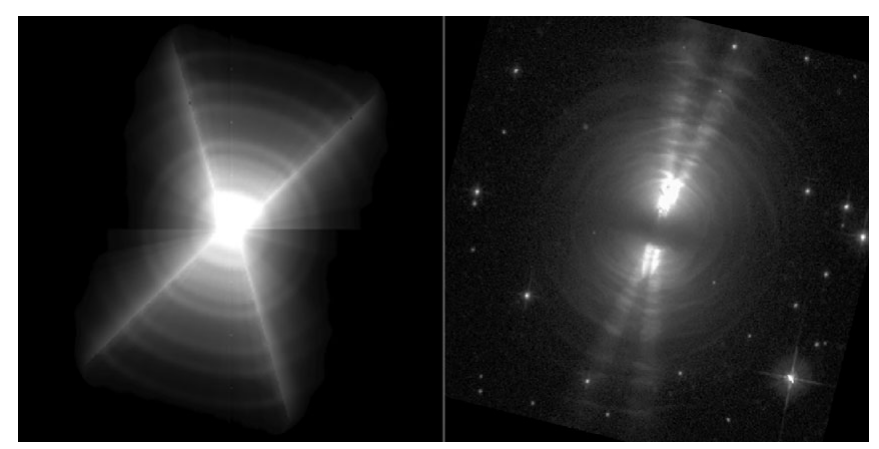

Figure 2. Left: SHAPE model inclined by $20^{\circ}$. Right: image of the Egg Nebula (Sahai et al. 1998). When rotated the ladder rungs become concentric arcs and the bright knots disappear.

appear to connect the ladder rungs to the walls of the nebula are also an optical effect. The etchings of the density enhancements left on the wall of the bi-cone are seen edge on. We see through more of the material at the tips of the ring and therefore they appear brighter, producing $\mathrm{t}$ he observed knots. The walls of the bi-cone are not completely opaque, and light from the star can penetrate into the dusty halo illuminating some of the dust. Outside the cavity, the dust remains in a spherical geometry and hence we see "extensions" from the walls that seem to flow backward. To demonstrate the illusion of the "ladder rungs" and the knots, we present the model inclined at $20^{\circ}$ in Figure 2. As the inclination increases, we clearly see the ladder structure give way to the more commonly observed concentric arcs (Kwok et al. 1998) and the knots disappear.

\section{References}

Cohen, M., et al. 1975, ApJ, 196, 179

Cohen, M., et al. 2004, AJ, 127, 2362

Kwok, S., Su, K., \& Hrivnak, B. 1998, ApJ, 501, L117

Sahai, R., et al. 1998, ApJ, 493, 301

Steffen, W., et al. 2011, IEEE Transactions on Visualization and Computer Graphics, 17, 454 